\title{
THEORETICAL STUDIES OF ATOMIC TRANSITIONS
}

\author{
Progress Report \\ Period: April 1, 2002 - March 31, 2005 \\ Charlotte FROESE FISCHER \\ Research Professor of Computer Science \\ Box 1697, Vanderbilt University \\ Nashville, Tennessee 37235 USA \\ Phone: (615) 322-2796; \\ Email: Charlotte.F.Fischer@Vanderbilt.edu \\ http://www.vuse.vanderbilt.edu/ ${ }^{\sim} \mathrm{cff} / \mathrm{cff} . \mathrm{html}$
}

July 8, 2005

Prepared for the U.S. Department of Energy under grant number DE-FG02-97ER14761 


\section{Research Progress Report}

Research during the last year of my grant and its two year, no-cost extension, continued to concentrated on the development and application of two theoretical models for atomic structure calculations, in general, and transition probabilities, in particular:

1. non-relativistic calculations where relativistic effects are included through the Breit-Pauli approximation which captures the lowest-order corrections, and

2. fully relativistic calculations based on a multconfiguration Dirac-Hartree-Fock (MCDHF) theory which may include also QED corrections and the effect of the finite size of the nucleus.

Variational configuration interaction methods were used in both cases where the wavefunction expansion is in terms of configuration state functions constructed from one-electron orbitals. Though the theories underlying these two formalisms are very different, computationally they are similar except for the fact that the Dirac-Hartree-Fock approach is considerably more computationally intensive.

In each case, the correlation interactions within a many-body system need to be accounted for in an accurate calculation. In many-body perturbation theory for relatively simple systems such as one or two-electrons outside closed shells, these are represented by classes of diagrams. In our work, where we wish to deal with arbitrarily complex systems, we use rule-based methods instead which are similar to those first introduced in quantum chemical applications. These rules are applied to orbital sets which are allowed to increase in size in a systematic fashion in order to determine convergence of an atomic property. In order to deal with more complex cases to higher accuracy, the codes for the two models have both been adapted to parallel execution using MPI. Some problems still remain with the MCDHF version but we hope to get those under control soon.

Three questions have motivated our research:

1. What rules are needed to adequately account for the effect of correlation on an atomic property of a many-electron system? Classes of correlation corrections have been identified - valence correlation (correlation among outer electrons), core-valence (representing the polarization of the core by outer electrons), and core-core correlation. For transition probabilities, One of the big advances in terms or accuracy has been the realization that outer or valence correlation alone may not be enough - core valence can have an appreciable effect at the 1-5\% level, and in some cases also correlation in the core.

2. When is the simpler Breit-Pauli calculation adequate? Or should the Dirac-Hartree-Fock methods always be used because the underlying physics theory is more correct? For light atoms, transition data depends on details of the computational procedure. How can reliable data be obtained?

A list of publications that appeared in since 2001 are given in the Appendix I. Not listed are posters presented at numerous conferences.

Postdocs and students supported are listed in Appendix II

\section{Breit-Pauli calculations for Be-like to Ar-like spectra}

For many years we have investigated isolated transitions or multiplets. Our understanding of the application of the Breit-Pauli method has reached the stage where "spectrum" calculations seemed feasible. By this we mean, wave function calculations for all the levels up to a certain region of 
the spectrum. For complex spectra, it became necessary to implement a feature in the DiracHartree-Fock of optimizing on an energy functional that is a weighted linear combination of energy expressions. This allowed us to obtain a basis that simultaneously gave good descriptions of the different terms. This was useful in that our current Breit-Pauli code assumes one common orbital basis. With this code, modified for parallel execution, we have performed calculations for Be-like (4 electrons) to Ar-like (18 electron) atoms and ions, often for nuclear charges up to $\mathrm{Z}=30$. The results for Be-like to Ne-like have been published [1] and Na-like to Ar-like has been accepted [2].

\section{The MCHF/MCDHF Database}

Large amounts of data are generated. By computing all transitions between levels, we are able to produce tables of energy-levels with lifetimes and tables of transition probabilities. These are posted on the web at

http://atoms.vuse.vanderbilt.edu

Initially, only the tables were posted but more recently, a search capability was added that allows the user to select the information to be displayed. At the same time, the system compares the computed energy levels with levels based on observation as displayed in the NIST Atomic Energy Level Database [3]. This information as well as the discrepancy in gauges for electric dipole transitions, provides some indicators of accuracy. At the same time, a number of errors in the classification of levels have been found.

Our database is part of the GENIE search engine (http://www-amdis.iaea.org/GENIE/) supported by the International Atomic Energy Agency (IAEA).

\section{Cases of Special interest}

In addition to the systematic calculation of transition probabilities for iso-electronic sequences, a number of calculations have been carried out in collaboration with scientists from a variety of fields.

1. Experimentalists interested in long lived states for which measurements can be made using the Stockholm storage ring have been investigating lifetimes of negative ions [4]. A number of their measurements have been confirmed.

2. Astrophyisicsts were interest in transition probabilities between levels of $3 d^{5}$ in Fe IV [5]. Earlier calculation for only a few transitions have been extended to all sixteen LS terms and 37 levels. These are the most accurate levels to date.

3. As a first step towards the study of time reversal and parity violation, the hyperfine properties of the ${ }^{3} D_{2}$ in radium as well as the transition probability for the $7 s 7 p^{3} P_{1}-7 s 6 d^{3} D_{2}$ transition were investigated using the Dirac-Hartree-Fock theory [6].

\section{$5 \quad$ Future activities}

Several projects have not been completed.

1. The atomic structure codes - non-relativistic, Breit-Pauli, and Dirac-Hartree-Fock - are in the process of being documented in preparation for submission to Computer Physics Communications. Both serial and parallel versions are available. 
2. The present variational codes are all based on the numerical solution of differential equations. A feature of differentail equations is that they are plagued by a series of special cases. Through the use of a B-spline expansion of orbitals, the variational procedure can be applied to the energy functional in terms of such an expansion and leads to non-linear equations of eigenvalue type for which can be solved using carefully developed numerical LAPACK routines. This application will be developed as time permits.

\section{References}

[1] C. Froese Fischer and G. Tachiev, Atomic Data and Nuclear Data Tables 871 - 184 (2004).

[2] C. Froese Fischer, G. Tachiev, and A. Irimia Atomic Data and Nuclear Data Tables (accepted).

[3] NIST Atomic Spectra Database Version 3 (http://physics.nist.gov/asd/).

[4] A. Ellmann, P. Schef, P. Lundin, P. Royen, S. Mannervik, K. Fritoff, P. Andersson, D. Hanstorp, C. Froese, Physical Review Letters 92253002 (2004).

[5] C. Froese Fischer and R. H. Rubin, Mon. Not. Royal Astron. Soc. 355 461-474 (2004): erratum ibid 3551400 - 1400 (2004).

[6] J. Bieron, C. Froese Fischer, S. Fritzsche, and K. Pachucki, J. Phys. B: Atom. Molec. Phys. 37 L305-L311 (2004). 


\section{Journal Publications}

\section{APPENDIX I}

1. "Breit-Pauli energy levels and transition rates for nitrogen-like and oxygen-like sequences" G. Tachiev and C. Froese Fischer Astron. Astophys. 385 716-723 (2002).

2. "Landé g factors for $2 p^{4}\left({ }^{3} P\right) 3 p$ and $2 p^{4}\left({ }^{3} P\right) 3 d$ states of Ne II" C. Froese Fischer and P. Jönsson

J. Mol. Struct. (THEOCHEM) 537, 55-62 (2001).

3. "Resonance transition energies and oscillator strengths in Lutetium and Lawrencium" Y, Zou and C. Froese Fischer Phys. Rev. Letters 88183001 (2002).

4. "Photodetachment of He- $1 \mathrm{~s} 2 \mathrm{~s} 2 \mathrm{p}^{4} \mathrm{P}^{o}$ in the region of the 1s threshold" O. Zatsarinny, T. W. Gorczyka, and C. Froese Fischer, J. Phys. B: Atom. Molec. Phys. 35416 - 4178 (2002).

5. "Hyperfine structure calculations of excited levels in neutral scandium" J. Bieron, C. Froese Fischer, and M. Godefroid, J. Phys. B: Atom. Molec. Phys. 353337 - 3345 (2002).

6. "Oscillator strengths for transitions of high-lying excited states of carbon" O. Zatsarinny and C. Froese Fischer J. Phys. B: Atom. Molec. Phys. 354669 - 4683 (2002).

7. "Allowed and spin-forbidden electric dipole transtions in Ca I" C. Froese Fischer and G. Tachiev Phys. Rev. A 68012507 (2003).

8. "Breit-Pauli energy levels, lifetimes, and transition probabilities for the beryllium-like to neon-like sequences" C. Froese Fischer and G. Tachiev, Atomic Data and Nuclear Data Tables 871 - 184 (2004).

9. "Breit-Pauli and Dirac-Hartree-Fock energy levels and transition probabilities in neutral argon" A. Irimia and C. Froese Fischer, J. Phys. B: Atom. Molec. Phys. 371659 - 1672 (2004).

10. "Radiative lifetime of a bound excited state of Te-" A. Ellmann, P. Schef, P. Lundin, P. Royen, S. Mannervik, K. Fritoff, P. Andersson, D. Hanstorp, C. Froese, Physical Review Letters 92253002 (2004).

11. "The Landé g-factor in atomic spectroscopy" C. Froese Fischer, P. Jnsson, and G. Tachiev, Molecular Physics 1021177 - 1184 (2004).

12. "Lifetime and hyperfine structure of the ${ }^{3} D_{2}$ state of radium" J. Bieron, C. Froese Fischer, S. Fritzsche, and K. Pachucki, J. Phys. B: Atom. Molec. Phys. 37 L305-L311 (2004).

13. "Breit-Pauli energy levels, transition probabilities and lifetimes for $3 d^{5}$ levels in Fe IV of astrophysical interest"

C. Froese Fischer and R. H. Rubin,

Mon. Not. Royal Astron. Soc. 355 461-474 (2004): erratum ibid 3551400 - 1400 (2004).

14. "Angular integrations using symbolic state expansions"

C. Froese Fischer and D. Ellis,

Lithuanian J. Physics 44121 - 134 (2004). 
15. "Breit-Pauli oscillator strengths, lifetimes and Einstein A-coefficients in singly ionized sulphur" A. Irimia and C. Froese Fischer, Physica Scripta 72172 - 184 (2005).

16. "Breit-Pauli lifetimes and transition probabilities for Si I"

C. Froese Fischer,

Physical Review A 71042506 (2005).

17. "Relativistic calculations for highly charged ions"

C. Froese Fischer,

Nucl. Inst. Meth. Phys. Res. B: 235100 - 104 (2005).

18. "Relativistic energy levels, lifetimes, and transition probabilities for the Na-like to Ar-like sequences" C. Froese Fischer, G. Tachiev, and A. Irimia, Atom. Data Nucl. Data Tables (accepted).

19. "Oscillator strength calculations in neutral technetium" P. Palmeri, C. Froese Fischer, J.-F. Wyart and M. Godefroid, Mon. Not. Royal Astron. Soc. (accepted). 
1. Dr. Baek Il Nam, from South Korea, was supported for a year beginning September 21, 2001

2. Dr. Georgio Tachiev, who had been a postdoc for more than three years beginning in 1997 and who designed and implemented our database, made visits during 2003 and 2004.

3. A graduate student was employed for the eight months of January - August of 2004. 